\title{
EVN observations of weak blazars
}

\section{Franco Mantovani*}

Max-Planck-Institut für Radioastronomie, Bonn, Germany

Istituto di Radioastronomia INAF, Bologna, Italy

E-mail: fmantovani@mpifr-bonn.mpg.de - fmantovani@ira.inaf.it

\section{Marco Bondi}

Istituto di Radioastronomia INAF, Bologna, Italy

E-mail: bondieira.inaf.it

\section{Karl-Heinz Mack}

Istituto di Radioastronomia INAF, Bologna, Italy

E-mail: mackeira.inaf.it

\section{Walter Alef}

Max-Planck-Institut für Radioastronomie, Bonn, Germany

E-mail: alef@mpifr-bonn.mpg.de

\section{Eduardo Ros}

Max-Planck-Institut für Radioastronomie, Bonn, Germany

Observatorio Astronòmico, Universitat de València, València, Spain

Departament d'Astronomia i Astrofísica, Universitat de València, València, Spain

E-mail: ros@mpifr-bonn.mpg.de

\section{Anton Zensus}

Max-Planck-Institut für Radioastronomie, Bonn, Germany

E-mail: azensus@mpifr-bonn.mpg • de

We undertook a survey with the European Very Long Baseline Interferometry Network at $5 \mathrm{GHz}$ of a complete sample of weak blazars, aiming to a follow-up comparison between high- and lowpower samples of blazars. We observed 87 sources extracted from the "Deep X-ray Radio Blazar Survey". All of the sources observed were detected. Point-like sources on milli-arcsecond scale are found in 39 cases, 45 show core-jet structure, and 3 possibly have triple structure. According to their spectral indices, 56 sources show a flat or inverted spectral index. They can be considered blazars. Twenty-nine sources show a steep spectrum or a spectrum peaking at a frequency around $1-2 \mathrm{GHz}$. Fourteen flat spectrum plus 1 steep spectrum sources are also $\gamma$-ray emitters detected by the Fermi Gamma-ray Space Telescope. About 50\% of them are BL Lacs. The DXRBS containes about $13 \%$ of BL Lacs in total. This supports the finding that BL Lacs are more preferably detected by Fermi. We confirm the correlation found between the source core flux density and the $\gamma$-ray photon fluxes down to fainter flux densities. We found that weak blazars are also weaker $\gamma$-ray emitters compared to bright blazars.

12th European VLBI Network Symposium and Users Meeting

7-10 October 2014

Cagliari, Italy

${ }^{*}$ Speaker. 


\section{Introduction}

Less than $10 \%$ of all the Active Galactic Nuclei (AGN) are flat-spectrum radio quasars (FSRQs) and BL Lacertae objects (BLLacs). Together, these two classes of objects are named blazars. They are well known $\gamma$-ray sources as shown by the Second Fermi-LAT catalogue (2FGL, [15]). The connection between relativistic Doppler boosting and $\gamma$-ray emission in AGN was suggested by many authors ([1], [6], [7], [12], [20]). Bright blazars are the target of many programmes for both single-dish and interferometric observations in the radio band, like the MOJAVE programme which regularly observes with the Very Long Baseline Array objects with flux density $>1.5 \mathrm{Jy}$ [12]. To increase the knowledge on blazars a deeper sample has been assembled by [17] and by [8], the "Deep X-ray Radio Blazar Survey" (DXRBS), currently the faintest and largest blazar sample with nearly complete optical identifications. We started a follow up investigation on the DXRBS objects, aiming to verify their spectral index classification and making the first milli-arcsecond resolution observations, for a comparison between high- and low-power samples of blazars. First we made simultaneous multi-frequency flux density measurements with the Effelsberg 100-m telescope to properly define their spectral index [14], then we undertook a survey with the European Very Long Baseline Interferometry Network (EVN) ${ }^{1}$, subject of this contribution.

\section{Observations and data reduction}

The observations were made with the EVN at $5 \mathrm{GHz}$ in several sessions along the period October 2009 - May 2013. Each source was tracked for 4 to 5 scans each 6 minutes long at different hour angles. About 30 minutes of observing time per source allow to reach a rms noise of $\sim 0.1 \mathrm{mJy} / \mathrm{beam}$ in the best case.

The recorded data were correlated at the DiFX software correlator [2] of the Max-PlanckInstitut für Radioastronomie (Bonn, Germany) and those in real time (e-EVN) were correlated at the Joint Institute for VLBI in Europe (Dwingeloo, The Netherlands). The correlator output was calibrated in amplitude and phase using $A I P S^{2}$ and imaged using DIFMAP ${ }^{3}$ [19].

\section{Results}

All of the 87 sources observed have been detected. Among them, point-like sources are found in 39 cases, 45 show a core-jet structure, and 3 possibly are triple sources. Examples of sources classified as above are presented in Fig. 1. The classification of sources based on their milliarcsecond (mas) structure and on the spectral indices [14] showed that 56 objects are blazars with flat or inverted spectral indices, 10 objects are Steep Spectrum Radio Quasars (SSRQs), 10 can be classified as Compact Steep-spectrum Sources (CSSs). Six more objects show convex spectra and they are GigaHz-Peaked Sources (GPSs). We also detected 2 NLRGs. Two BL Lacs show a steep spectrum and one a convex spectrum, however their optical classification is still questionable.

\footnotetext{
${ }^{1}$ The European VLBI Network is a joint facility of European, Chinese, South African and other radio astronomy institutes funded by their national research councils.

${ }^{2}$ AIPS is the NRAO's Astronomical Image Processing System

${ }^{3}$ DIFMAP is part of the Caltech VLBI software Package
} 

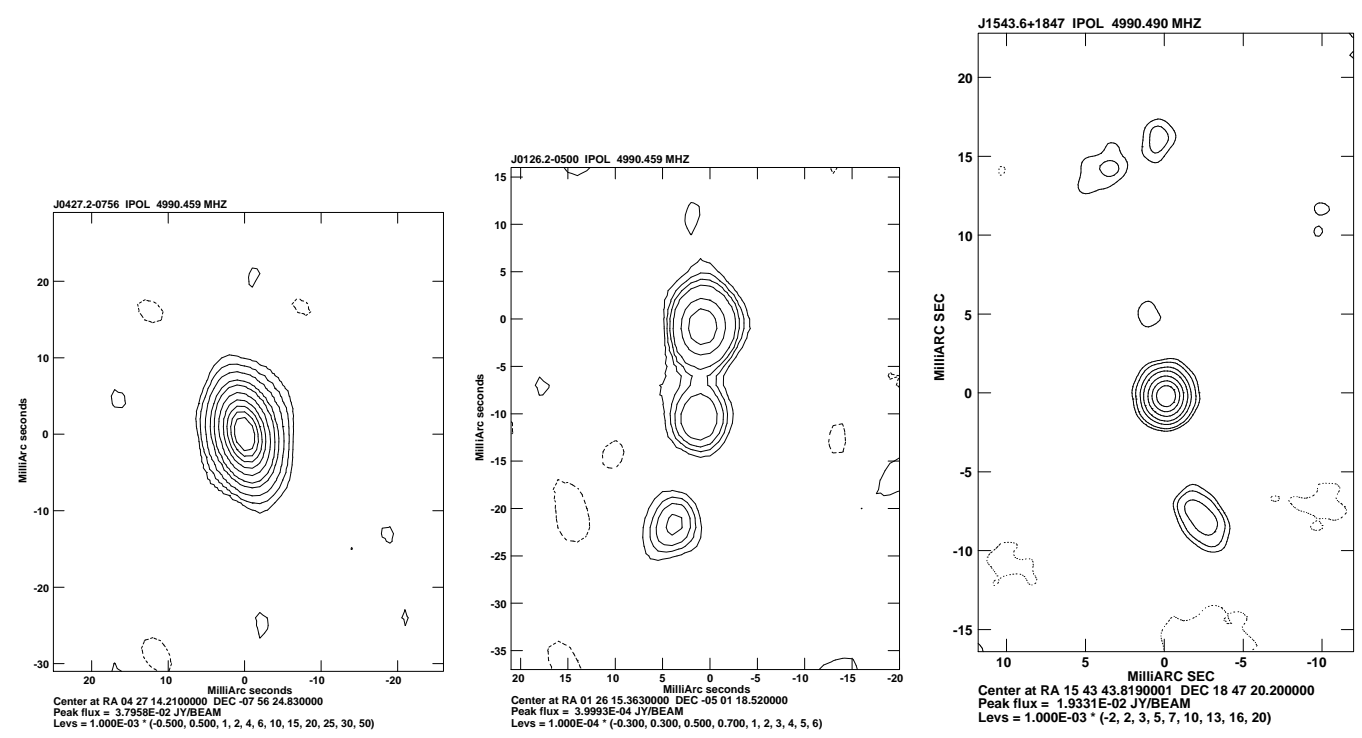

Figure 1: Total-intensity images of the point-like source J0427.2-0756, the core-jet source J0126.2-0500, and the triple source J1543.6+1847. The convolution beam is $7.6 \times 4.3$ mas at $14.8 \mathrm{deg}, 6 \times 6$ mas, and $2.5 \times 2.0$ mas at $5.4 \mathrm{deg}$, respectively.

\section{Derived parameters}

Source parameters derived from the present EVN observations are as follows. The total flux density distribution has a median value $\langle S\rangle=44_{-10}^{+16} \mathrm{mJy}{ }^{4}$. Sixty-eight out of 87 sources show a total flux density $\leq 150 \mathrm{mJy}$. The ratio between the EVN flux density at $5 \mathrm{GHz}$ from the present observations to the Effelsberg flux density previously measured by [14], shows a median value $\langle R\rangle=0.36 \pm 0.08$ suggesting the presence of more extended structures around the compact detected objects. The vast majority of sources show $\mathrm{R}<1$, while only 11 sources have $\mathrm{R}>1$. Note that the Effelsberg and the EVN flux density measurements were taken in different periods. The measurements of both flux density and size of the core at mas scale, together with the available red-shift [17] [8], allowed us to figure out lower limits of the brightness temperature $\mathrm{T}_{b}$ which ranges between $10^{7} \mathrm{~K}$ and $10^{12} \mathrm{~K}$. Thirteen sources show $\mathrm{T}_{b}>10^{11} \mathrm{~K}$. Only one object, namely J0937.1+5008, has $\mathrm{T}_{b}$ close to $10^{12} \mathrm{~K}$.

\section{DXRBS sources associated to Fermi objects}

We search for any possible association between the DXRBS sources and the objects detected by Fermi listed in the 2FGL catalogue. Since all the DXRBS objects observed with the EVN were detected, we assume that the DXRBS sources in the Southern sky observed with ATCA are also compact objects detectable with interferometric observations at milli-arcsecond resolution. The associations are listed in Table 1 where we have in column 1 the 2FGL name; in 2 the optical

\footnotetext{
${ }^{4}$ In a set of $\mathrm{n}$ values, the median value is the $n / 2$ ranked value. The statistical errors associated to it is given taking the median values $\pm \sqrt{n}$ ranked value.
} 


\begin{tabular}{llcl}
\hline $\begin{array}{l}\text { EVN Objects } \\
\text { Name 2FGL }\end{array}$ & O.I & $\begin{array}{c}\text { Radio- } \gamma \text { Sep. } \\
\text { [arcmin }]\end{array}$ & Association \\
\hline J0205.0+1514 & SSRQ & 2.7 & 4C15.05 \\
J0509.9+1802 & FSRQ & 1.7 & PKS 0507+17 \\
J0847.2+1134 & BLLac & 0.8 & RX J0847.1+1133 \\
J0937.6+5009 & FSRQ & 7.4 & GB6 J0937+5008 \\
J1010.8-0158 & FSRQ & 4.1 & PKS 1008-01 \\
J1204.3-0711 & BL Lac & 1.8 & 1RXS J120417-070959 \\
J1231.7+2848 & BLLac & 0.4 & B2 1229+29 \\
J1332.7+4725 & FSRQ & 10.4 & B3 1330+476 \\
J1656.5+6012 & FSRQ & 4.5 & 87GB 165604.4+601702 \\
\hline ATCA Objects & & & \\
\hline J0448.6-2118 & FSRQ & 9.9 & PKS 0446-212 \\
J0449.4-4350 & BL Lac & 1.1 & \\
J1610.6-4002 & FSRQ & 6.1 & PMN J1610-3958 \\
J1936.8-4721 & BL Lac & 2.0 & PMN J1936-4719 \\
J2258.8-5524 & BL Lac & 9.1 & PMN 2258-5526 \\
J2330.6-3723 & BLLac & 0.5 & PKS 2327-376 \\
\hline
\end{tabular}

Table 1: Association between DXRBS and Fermi objects

identification; in 3 the separation between the radio and the $\gamma$ object; in 4 the name of the associated radio source as suggested in $2 \mathrm{FGL}$.

Considering all the radio- $\gamma$ associations, about 50\% of the objects listed in Table 1 are classified as BLLac. Keeping in mind that the DXRBS containes about $13 \%$ of BL Lac objects in total, this confirms that these objects are more preferably detected by Fermi than FSRQs, as found in [1].

\section{Discussion}

We made the first mas resolution observations of a complete sample of blazars selected from the faintest DXRBS only applying the criteria of a cut in declination at $>-10 \mathrm{deg}$. The sample is formed by 87 target objects, $\sim 13 \%$ of which are BL Lacs. All sources were detected confirming they actually are compact objects. The 56 true blazars we found show a median flux density $\left\langle S_{t o t}\right\rangle=75_{-27}^{+15} \mathrm{mJy}$. Of them, 42 show a flux density $\mathrm{S}<150 \mathrm{mJy}$ at $5 \mathrm{GHz}$. They potentially represent the appropriate sample for a comparison between faint- and bright- blazar samples.

One of the most extended sample of bright flat spectrum blazars is the MOJAVE programme ([9], [10]) regularly observed with the Very Long Baseline Array ${ }^{5}$. It includes all the objects with VLBA flux density exceeding $1.5 \mathrm{Jy}$ at $15 \mathrm{GHz}$, declination $>-30 \mathrm{deg}$, plus all known AGNs above declination $>-20$ deg detected by Fermi and with compact flux density of at least $100 \mathrm{mJy}$ at $15 \mathrm{GHz}$. A highly significant correlation was found by [18] analysing a sample of 183 LAT-detected

\footnotetext{
${ }^{5}$ The VLBA is a facility of the National Science Foundation operated by The National Radio Astronomy Observatory under cooperative agreement with the Associated Universities, Inc.
} 


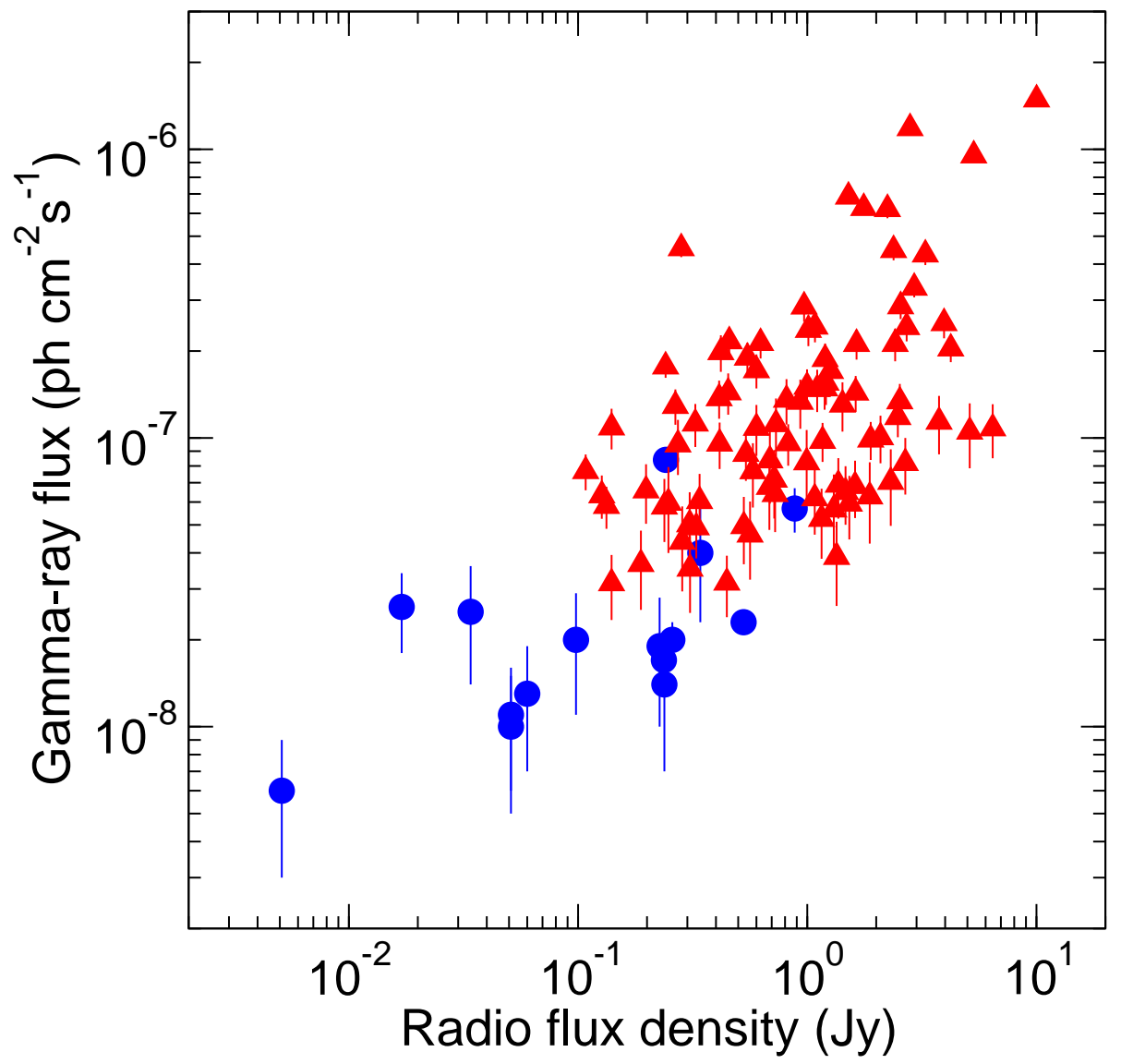

Figure 2: Integrated 0.1-100 GeV Fermi LAT photon flux vs. $15 \mathrm{GHz}$ VLBA core flux density (red triangles) as in Figure 2 of [18], complemented with data pairs from the present work (blue circles).

AGN between the source core flux density at $15 \mathrm{GHz}$ and the integrated $0.1-100 \mathrm{GeV} \gamma$-ray photon fluxes taken from the Fermi 1FGL. Since the correlation becomes weaker if the jet flux density is instead used, they suggest the core as the site of the $\gamma$-ray emission. Furthermore, they suggest that the region where most of $\gamma$-ray photons are produced is located within the compact opaque parsecscale core, with a typical distance between the $\gamma$-ray production region and the $15 \mathrm{GHz}$ radio core of $\sim 7 \mathrm{pc}$. A similar distance was recently also found by [5]. The presence of this correlation in this highly variable population further suggests that Doppler beamed sources are preferably detected in $\gamma$, and that Doppler factors of the individual jets are not changing substantially over time [18].

Most of the sources associated with Fermi 2FGL objects we observed with the EVN reaches a much lower level in flux densities at $5 \mathrm{GHz}$. The present sample of weak blazars is for the time being missing the monitoring programme needed to search for structural changes and for evaluating Doppler factors. However, the existing mas resolution observations allowed us to derive useful parameters which can be used to complement previous findings in bright blazars. The plot of Fig. 2 by [18] can be updated with new data pairs. We assume that the core flux density at $15 \mathrm{GHz}$ for sources in Table 1 is close to what we measured at $5 \mathrm{GHz}$ since they have a flat spectral index. The integrated $\gamma$-ray photon flux of the associated Fermi objects was evaluated fitting the full band (100 MeV-100 GeV) from the 2FGL catalogue [15]. The updated plot is shown in Fig. 2. 
Taking into account the uncertainties in the data pairs we added, the correlation is clearly confirmed towards weaker fluxes. We also found that weak blazars are also weaker $\gamma$-ray emitters compared to bright blazars, which may indicate that we may not need to invoke the external Compton model [3] to explain the apparent lack of faint $\gamma$-ray emitters in Fig. 2 as previously suggested by [11].

Finally, about half of the steep or convex spectrum sources found, show linear sizes $\leq 20 \mathrm{kpc}$ and power $P_{1.4 \mathrm{GHz}}>10^{25} \mathrm{~W} \mathrm{~Hz}^{-1}$. They can be classified as CSSs or GPSs, i.e., young radio sources with ages $<10^{7} \mathrm{yr}$ [4]. Their X-ray luminosity obtained by ROSAT observations ([17], [8]) is $10^{43}-10^{46} \mathrm{ergs} \mathrm{sec}^{-1}$ with a median value of $\left\langle L_{\mathrm{X}}\right\rangle \sim 7_{-3}^{+26} \times 10^{44} \mathrm{ergs} \mathrm{sec}^{-1}$, a bit lower compared to the luminosity of $10^{45}-10^{46} \mathrm{ergs} \mathrm{sec}^{-1}$ reported for CSSs by [16]. Any difference in X-ray luminosity between CSS and GPS quasars looks not significant. The column densities of hydrogen $\mathrm{nH}$ are of the order of the Galactic $\mathrm{nH}$ column density suggesting that CSS and GPS quasars are not obscured by large column of cold gas surrounding the nuclei.

\section{References}

[1] Ackermann, M., Ajello, M., Allafort, A. et al. 2011 AJ, 741, 30

[2] Deller, A.T., Brisken, W.F., Phillips, C.J. et al. 2011 PASP, 123, 275.

[3] Dermer, C.D. 1995, ApJ, 446, L63

[4] Fanti, R., Fanti, C., Schilizzi, R.T. et al. 1990 A\&A, 231,333

[5] Fuhrmann, L.; Larsson, S.; Chiang, J. et al. 2014 MNRAS 441, 1899

[6] Kellermann, K.I., Lister M.L., Homan D.C. et al. 2004 ApJ, 609, 539

[7] Kovalev, Y.Y., Kellermann, K.I., Lister, M.L. et al. 2005 AJ, 130, 2473

[8] Landt, H., Padovani, P., Perlman, E.S. et al. 2001 MNRAS, 323, 757

[9] Lister, M.L., Homan, D.C. 2005 AJ, 130, 1389

[10] Lister, M.L., Cohen, M.H., Homan, D.C. et al. 2009 AJ, 138, 1874

[11] Lister, M.L. "Fermi meets Jansky - AGN in Radio and Gamma Rays “, T. Savolainen, E. Ros, R.W. Porcas, \& J.A. Zensus Eds. (Bonn, MPIfR) 159

[12] Lister, M.L., Aller, M., Aller, H. et al. 2011 ApJ, 742, 27

[13] Mantovani, F., Mack, K.-H., Montenegro-Montes, F.M. et al. 2009 A\&A, 502, 61

[14] Mantovani, F., Bondi, M., \& Mack, K.-H. 2011 A\&A, 533, 79

[15] Nolan, P.L., Abdo, A.A., Ackermann, M. et al. 2012 ApJS, 199, 31

[16] O’Dea, C. 1998 PASP, 110, 493

[17] Perlman, E.S., Padovani, P., Giommi, P. et al. 1998 AJ, 115, 1253

[18] Pushkarev, A.B., Kovalev, Y.Y., \& Lister, M.L. 2010 ApJ, 722, L11

[19] Shepherd, M.C., Pearson, T.J., \& Taylor, G.B. 1995, BAAS, 26, 305

[20] Taylor, G.B., Healey, S.E., Helmboldt, J.F. et al. 2007 ApJ, 671, 1355 